\title{
Outage Probability Analysis of WPT Systems with Multiple-Antenna Access Point
}

\author{
Khaled M. Rabie, Bamidele Adebisi and Matjaz Rozman \\ School of Engineering, Division of Electrical Engineering \\ Manchester Metropolitan University, Manchester, UK, M15 6BH \\ Emails: \{k.rabie@mmu.ac.uk; b.adebisi@mmu.ac.uk; matjaz.rozman@mmu.ac.uk\}
}

\begin{abstract}
This paper analyzes the performance of a multiple-antenna access point (AP) system with an energy-constrained single-antenna destination node in various Bernoulli-Gaussian impulsive noise environments. More specifically, we deploy the harvest-then-transmit protocol where communication is accomplished over two distinct phases: i) power transfer phase (down-link), ii) information transmission phase (up-link). In this respect, an analytical expression for the ergodic outage probability is derived and validated with Monte Carlo simulations. Results have shown that increasing the source transmit power or/and the number of AP antennas will minimize the ergodic outage probability. It is also presented that careful selection of the energy harvesting time is important to enhance the system performance.
\end{abstract}

Index Terms-Energy harvesting, Bernoulli-Gaussian (BG) impulsive noise, harvest-then-transmit protocol, ergodic outage probability, wireless power transfer (WPT).

\section{INTRODUCTION}

$\mathbf{E}$ NERGY harvesting is an increasingly important technology to power-up low-energy consumption electronic devices, specially when replacing or recharging their batteries is dangerous such as in toxic environments, risky or inconvenient as in implantable medical device applications [1]. A more effective solution is to make such devices energy self-sufficient and self-sustaining by simply scavenging energy from surrounding environments. There are a variety of sources from which energy can be harvested such as solar energy, kinetic energy, thermal energy, radio-frequency (RF) energy [2], etc. Although some applications such as implantable medical device applications find it challenging to secure a constant and perpetual energy source, many other applications can use harvested energy to supplement the battery which will, as well, reduce its size. An example of the former is energy-constrained wireless networks.

To overcome the problem of irregular flow of harvested energy, wireless power transfer (WPT), where devices' batteries can be recharged using wireless electricity, has been widely studied in the past years. These systems are also referred to as wireless-powered communication networks (WPCNs) [3], [4]. Particularly, RF signals has recently attracted significant research interest because it can concurrently carry information as well as energy signals, also known as simultaneous wireless information and power transfer (SWIPT), see e.g. [1], [5] and the reference therein. In these systems, receivers can process information and harvest energy from the same received signals. Several RF energy harvesting protocols have been reported in the literature with different degrees

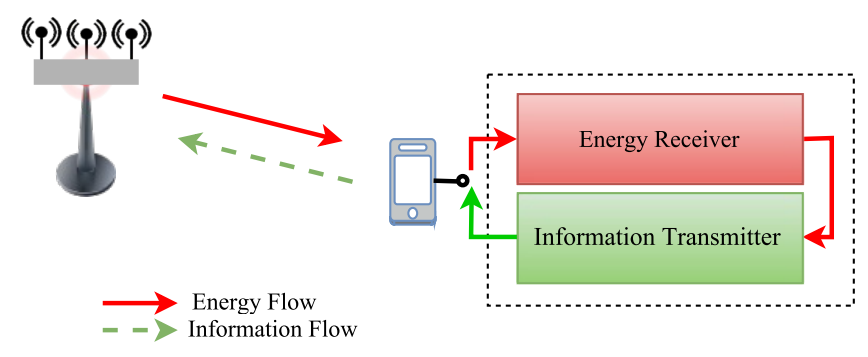

Figure 1: System model with down-link (DL) power transfer and uplink (UL) information transmission over BG impulsive noise channels.

of efficiency and complexity. For instance, in [6]-[8] the authors proposed the time switching relaying (TSR) and power splitting relaying (PSR) protocols. These protocols were studied in one-way single-antenna relaying networks where the relay harvests energy from the received signal and uses it to forward the information signal. The PSR protocol is based on splitting the received signal power into two parts, one for energy harvesting and the other for information processing. As for the TSR protocol, the relay switches between the energy harvester and the information receiver. In addition, the performance of energy harvesting systems in multiple-antenna relaying networks were investigated in [1], [9], [10].

All the aforementioned studies have assumed additive white Gaussian noise (AWGN). However, this assumption is not always valid because the noise can be non-Gaussian in many wireless communication environments as in cognitive radio [11], [12] and indoor radio channels [13], [14]. Unlike existing works, this paper is dedicated to analyze the ergodic outage probability performance of a WPCN system over non-Gaussian impulsive noise channels ${ }^{1}$. The system under consideration consists of a multiple-antenna AP and singleantenna destination node, see Fig. 1, in which end-to-end communication is accomplished over two phases as follows. The AP first broadcasts power signals during phase I (downlink) the energy of which is harvested by the destination node along with the impulsive noise signal power. In phase II (up-link), the destination uses the harvested energy and transmits its information to the AP. Although there are several impulsive noise statistical models used in the literature to evaluate communication performance over non-Gaussian

${ }^{1}$ Refer to [15] for the ergodic capacity analysis of a WPCN network in non-Gaussian impulsive noise environment. 
channels, such as Middleton Class-A, Class-B, Class-C [16], [17], Gaussian-Mixture noise [18] and Bernoulli-Gaussian (BG) noise [19], [20], the latter model will be adopted in this study. The proposed system performance is characterized in terms of the ergodic outage probability.

Therefore, the contribution of this paper is twofold. First, we derive an analytical expression for ergodic outage probability which is then validated with computer simulations. The second contribution resides in examining the impact of various system parameters on the system performance including the impulsive noise probability of occurrence, transmit source power and the number of AP antennas. Results have shown that increasing the source transmit power and/or the number of AP antennas will enhance the outage probability performance. In addition, it is presented that as the impulsive noise probability becomes higher, the performance worsens.

The rest of this paper is structured as follows. Section II describes the system model used in this study. In section III, we derive an analytical expression for ergodic outage probability. Numerical examples and simulation results are then presented and discussed in section IV. Finally, section $\mathrm{V}$ concludes the paper.

\section{System Mode}

As mentioned in the introduction, the system model under consideration consists of a multiple-antenna AP and one energy-constrained node separated by distance $d$ in an impulsive noise environment as shown in Fig. 1. The system deploys the well-known harvest-then-transmit protocol [8], the principle of which is illustrated in Fig. 2. It is assumed that the energy-constrained node has no fixed-power supply, i.e. it relies entirely on the energy harvested during phase I, and is equipped with a battery to store this energy. It is also assumed that all channel coefficients remain constant over the block time and vary independently and identically from one block to another according to Rayleigh distribution.

Noise at both the AP and destination node consists of two components, background noise and impulsive noise with variances $\sigma_{w}^{2}$ and $\sigma_{i}^{2}$, respectively. This noise is modeled here using the BG noise model (1) in which the background component, $n_{w}$, is considered complex Gaussian with zero mean and variance $\sigma_{w}^{2}$ whereas the impulsive component, $n_{i}$, is modeled as a BG random process [19], [21], [22]. Hence,

$$
n_{t}=n_{w}+n_{i}
$$

where $n_{t}$ is the total noise, $n_{i}=\mathrm{b} g, g$ is complex white Gaussian noise with mean zero and $\mathrm{b}$ is the Bernoulli process with probability mass function

$$
\operatorname{Pr}(\mathrm{b})= \begin{cases}p, & \mathrm{~b}=1 \\ 1-p, & \mathrm{~b}=0\end{cases}
$$

and $p$ is the impulsive noise probability of occurrence. The probability density function (pdf) of the total noise can therefore be given as

$$
P_{n_{t}}\left(n_{t}\right)=\sum_{m=0}^{1} p_{m} \mathcal{G}\left(n_{t}, 0, \sigma_{m}^{2}\right)
$$

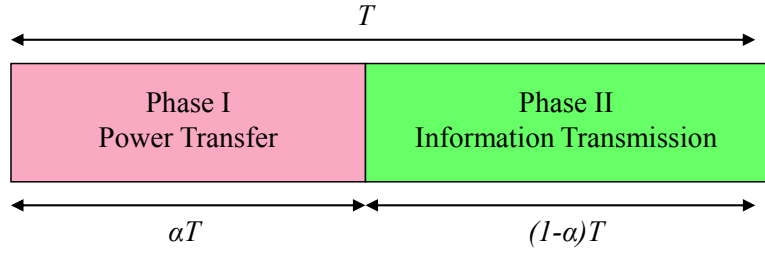

Figure 2: Harvest-then-transmit protocol used in this study.

where $p_{0}=1-p, p_{1}=p, \sigma_{0}^{2}=\sigma_{w}^{2}, \sigma_{1}^{2}=\sigma_{w}^{2}+\sigma_{i}^{2}$ and $\mathcal{G}($.$) is the Gaussian pdf given by \mathcal{G}\left(x, \mu, \sigma_{x}^{2}\right)=$ $\frac{1}{\sqrt{2 \pi \sigma_{x}^{2}}} \exp \left(-\frac{(x-\mu)^{2}}{2 \sigma_{x}^{2}}\right)$. The variances $\sigma_{w}^{2}$ and $\sigma_{i}^{2}$ define the input signal-to-background noise ratio (SBNR) and signal-toimpulsive noise ratio (SINR) as $\operatorname{SBNR}=10 \log _{10}\left(1 / \sigma_{w}^{2}\right)$ and $\operatorname{SINR}=10 \log _{10}\left(1 / \sigma_{i}^{2}\right)$, respectively. It is worthy mentioning that, unlike the background noise, the impulsive component is characterized by high instantaneous power and short duration, generally generated from electromagnetic and electronic equipments.

The end-to-end communication in this system is accomplished as follows. The AP first broadcasts energy signals during the first $\alpha T$ block time (phase I), where $0<\alpha<1$ is the energy harvesting time, while the energy-constrained node harvests this energy along that of background and BG impulsive noise in the surrounding. In the second time segment $(1-\alpha) T$ (phase II), using the harvested energy, the destination node transmits its information signal to the AP. Therefore, the received signal at the destination node during the power transfer phase can be given by

$$
\mathbf{y}_{D}=\sqrt{\frac{P_{s}}{d^{m}}} \mathbf{h}_{1} s+n_{D, w}+n_{D, i}
$$

where $P_{s}$ is the source transmit power, $m$ is the path loss exponent, $\mathbf{h}_{1}$ is the $1 \times N$ AP-to-destination channel vector, $s$ is the transmitted signal (energy-carrying signal) with zero mean and unit variance, $n_{D, w}$ and $n_{D, i}$ denote the background and impulsive noise components at the destination node, respectively. Now, the harvested energy at the destination can be expressed as

$$
E_{h}=\eta \alpha T \mathbb{E}\left[\left\|\mathbf{y}_{D}\right\|^{2}\right]
$$

while $\eta$ is the energy harvesting efficiency, $\mathbb{E}[$.$] and \|$. denote the expectation and Euclidean norm operators, respectively. Substituting (4) into (5) yields

$$
E_{h}=\eta \alpha T\left(\frac{P_{s}}{d^{m}}\left\|\mathbf{h}_{1}\right\|^{2}+\sigma_{D, w}^{2}+p \sigma_{D, i}^{2}\right)
$$

The transmitted power at the destination node during phase II can be simply written as

$$
P_{D}=\frac{E_{h}-E_{s c}}{(1-\alpha) T}
$$

where $E_{s c}$ is the energy consumption by the receiver for information processing. However, it is commonly assumed that $E_{s c}$ is negligible compared to the harvested energy, hence $E_{s c}=0$. Now, by substituting (6) into (7), we obtain 


$$
\mathbb{E}\left[\left|y_{S}\right|^{2}\right]=\underbrace{\frac{\eta \alpha}{(1-\alpha) d^{m}}\left(\frac{P_{s}}{d^{m}}\left|\mathbf{h}_{1} \mathbf{h}_{2}\right|^{2}+\left(\sigma_{D, w}^{2}+p \sigma_{D, i}^{2}\right)\left\|\mathbf{h}_{2}\right\|^{2}\right)}_{\text {Information Signal Part }}+\underbrace{N\left(\sigma_{S, w}^{2}+p \sigma_{S, i}^{2}\right)}_{\text {Noise Part }} .
$$

$$
P_{D}=\frac{\eta \alpha}{(1-\alpha)}\left(\frac{P_{s}}{d^{m}}\left\|\mathbf{h}_{1}\right\|^{2}+\sigma_{D, w}^{2}+p \sigma_{D, i}^{2}\right)
$$

Furthermore, the transmitted signal at the destination node can be written as

$$
x_{D}=\sqrt{P_{D}} x
$$

where $x$ is the information signal normalized as $\mathbb{E}\left[|x|^{2}\right]=$ 1. The information signal received at the AP in the second phase is given by

$$
y_{S}=\sqrt{\frac{P_{D}}{d^{m}}} x \mathbf{h}_{2}+\mathbf{n}_{S, w}+\mathbf{n}_{S, i}
$$

where $\mathbf{h}_{2}$ is the $N \times 1$ destination-to-AP channel vector and $\mathbf{n}_{S}$ is the $N \times 1$ noise vector at the AP. Substituting (8) into (10), we can express the expectation of (10) as in (11), shown at the top of the next page.

Assuming perfect knowledge of $p$ is available at the AP, the system capacity can be simply calculated as [23]

$$
C=(1-\alpha) \sum_{m=0}^{1} p_{m} \log _{2}\left(1+\gamma_{m}\right)
$$

where $\gamma_{0}=\gamma_{S}$ and $\gamma_{1}=\gamma_{S} / \beta, \beta=\left(1+\sigma_{S, i}^{2} / \sigma_{S, w}^{2}\right) ; \gamma_{S}$ is the SBNR at the AP which can be obtained from (11) as

$$
\gamma_{S}=\frac{\eta \alpha\left(\frac{P_{s}}{d^{m}}\left|\mathbf{h}_{1} \mathbf{h}_{2}\right|^{2}+\left(\sigma_{D, w}^{2}+p \sigma_{D, i}^{2}\right)\left\|\mathbf{h}_{2}\right\|^{2}\right)}{(1-\alpha) N d^{m} \sigma_{S, w}^{2}}
$$

\section{Outage Probability Analysis}

This section derives an analytical expression for the ergodic outage probability, $P_{\text {out }}$, which is defined as the probability that the capacity falling below a certain threshold value, denoted here as $C_{t h}$. Mathematically,

$$
P_{\text {out }}=\operatorname{Pr}\left[C<C_{t h}\right]
$$

Using (12), the ergodic outage probability, for high SNR approximation, can be written as

$$
\begin{aligned}
P_{\text {out }} & \simeq \operatorname{Pr}\left\{\sum_{m=0}^{1} p_{m} \log _{2}\left(\gamma_{m}\right)<\frac{C_{t h}}{(1-\alpha)}\right\} \\
& \simeq \operatorname{Pr}\left\{\log _{2}\left(\gamma_{S}\right)^{(1-p)}+\log _{2}\left(\frac{\gamma_{S}}{\beta}\right)^{p}<\frac{C_{t h}}{(1-\alpha)}\right\} \\
& \simeq \operatorname{Pr}\left\{\left(\gamma_{S}\right)^{(1-p)}\left(\frac{\gamma_{S}}{\beta}\right)^{p}<2^{\frac{C_{t h}}{(1-\tau)}}\right\} \\
& \simeq \operatorname{Pr}\{\gamma_{S}<\underbrace{\beta^{p} 2^{\frac{C_{t h}}{(1-\tau)}}}_{v}\}
\end{aligned}
$$

where $\gamma_{S}$ is given by (13). Now, let

$$
\begin{aligned}
a & =\eta \alpha P_{s} / d^{m}, \\
b & =\eta \alpha \sigma_{D}^{2}, \\
c & =(1-\alpha) N d^{m} \sigma_{S}^{2}, \\
X & =\frac{\left|\mathbf{h}_{1} \mathbf{h}_{2}\right|^{2}}{\left\|\mathbf{h}_{2}\right\|^{2}} \text { and } \\
Y & =\frac{1}{\left\|\mathbf{h}_{2}\right\|^{2}},
\end{aligned}
$$

we can rewrite (13) as

$$
\gamma_{S}=\frac{a X+b}{c Y}
$$

Substituting (17) into (15), and with some algebraic manipulation, we can obtain

$$
\begin{aligned}
P_{\text {out }} & =\operatorname{Pr}\left[X<\frac{v c Y-b}{a}\right] \\
& =1-\operatorname{Pr}\left[X>\frac{v c Y-b}{a}\right]
\end{aligned}
$$

The outage probability in (18) can now be calculated as

$$
P_{\text {out }}=1-\int_{0}^{\frac{b}{v c}} f_{Y}(z) d z-\int_{\frac{b}{v c}}^{\infty} \bar{F}_{X}\left(\frac{v c z-b}{a}\right) f_{Y}(y) d z
$$

where $f_{Y}($.$) is the pdf of Y$ and $\bar{F}_{X}($.$) is the complemen-$ tary commutative distribution function (ccdf) of the random variable $X$. Since $X$ has exponential distribution, its ccdf can be expressed as

$$
\bar{F}_{X}\left(\frac{v c z-b}{a}\right)=\exp \left(-\frac{(v c z-b) \lambda_{x}}{a}\right)
$$

On the other hand, $Y$ is shown to have inverse chi-square distribution and, therefore, its pdf can be simply given by [24]

$$
f_{Y}(z)=\frac{\exp \left(-\frac{1}{z}\right) z^{-(N+1)}}{\Gamma(N)}
$$

where $\Gamma($.$) is the Gamma function defined as$

$$
\Gamma(x)=\int_{0}^{\infty} \exp (-t) t^{x-1} d t
$$

Finally, the system outage probability can be obtained by substituting (20) and (21) into (19) to yield (23) which is further simplified in (24), both are shown at the top of the next page. Next, we present some numerical examples of these expressions. 


$$
\begin{aligned}
P_{\text {out }} & =1-\frac{1}{\Gamma(N)} \int_{0}^{\frac{b}{v c}} \exp \left(-\frac{1}{z}\right) z^{-(N+1)} d z-\frac{1}{\Gamma(N)} \int_{\frac{b}{v c}}^{\infty} \exp \left(-\frac{(v c z-b) \lambda_{x}}{a}-\frac{1}{z}\right) z^{-(N+1)} d z \\
& =1-\frac{1}{\Gamma(N)}\left(\Gamma\left(N, \frac{v c}{b}\right)+\int_{\frac{b}{v c}}^{\infty} \exp \left(-\frac{(v c z-b) \lambda_{x}}{a}-\frac{1}{z}\right) z^{-(N+1)} d z\right)
\end{aligned}
$$

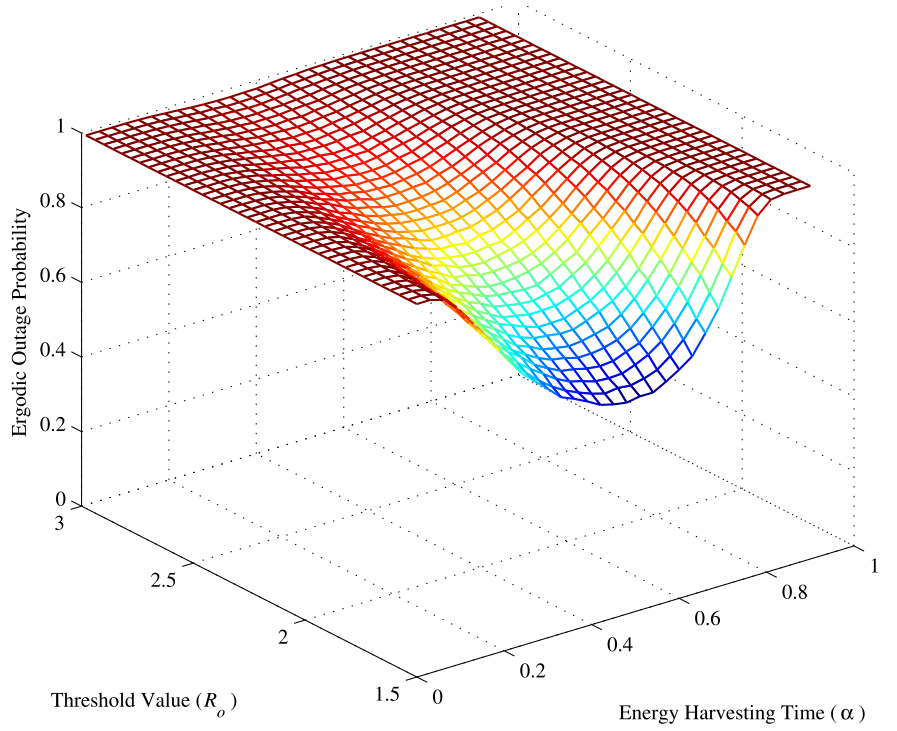

(a) $P_{s}=1$ watt.

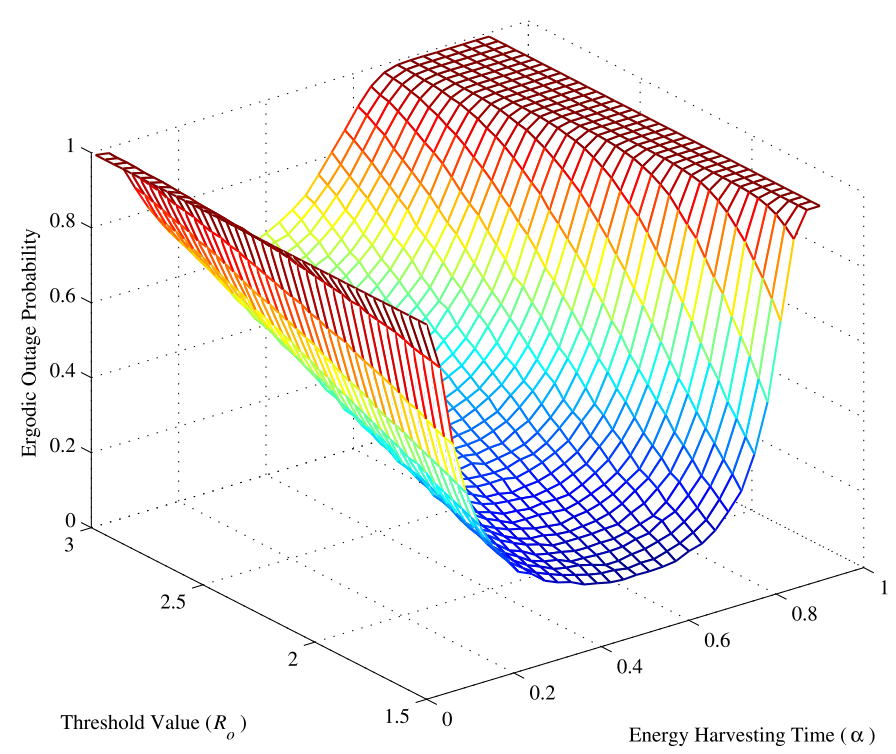

(b) $P_{s}=5$ watt.

Figure 3: 3D surface plots of the ergodic outage probability as a function of the energy harvesting time and threshold value for different transmit source power values.

\section{NumERICAL RESUlTS AND DisCUSSIONS}

This section presents some numerical examples of the ergodic outage probability expression derived above. Various system parameters and noise scenarios are investigated. Throughout this section, we use $\lambda_{x}=1, \eta=1$, and $d$ is chosen $1 m$ for simplicity. To begin with, we show in Figs. $3 \mathrm{a}$ and $3 \mathrm{~b} 3 \mathrm{D}$ surface plots for the ergodic outage probability as a function of energy harvesting time and outage threshold value for two different transmit source powers when SBNR $=25 \mathrm{~dB}, p=0.01$, SINR $=-10 \mathrm{~dB}, N=10, m=2.7$ and $P_{s}=1$ and 5 watt. A common observation in the two figures one can clearly see is that increasing the threshold value leads to worsening the outage probability. In addition, it is noticeable that in each case there exists an optimal energy harvesting time factor that minimizes the outage probability. Now, comparing the two 3D plots, it is apparent that as the transmit source power is increased, the probability is minimized. This is because of the fact that increasing the source power means more energy can be harvested during phase I at the destination, which, consequently, lowers the outage probability in the up-link at the AP.

Furthermore, Fig. 4 depicts the minimum achievable ergodic outage probability for several antenna numbers: $N=$ 1, 2, 4, and 16, with different impulsive noise probabilities: $p=0.01$ and 0.001 . The system parameters considered here are $\mathrm{SBNR}=20 \mathrm{~dB}, \mathrm{SINR}=-10 \mathrm{~dB}, m=2$ and $P_{s}=3$ watt. It should be mentioned that the analytical results are found by substituting the optimal energy harvesting time factor $\alpha^{*}$ into (24). The good agreement between the analytical and simulated results verify the accuracy of our analysis. It is clear from these results that the performance improves as the number of antennas in increased irrespective of the impulsive noise probability considered. It is also noticeable that as the noise probability becomes higher, the outage probability worsens which is intuitive. The final remark on these results is that when the threshold is very large, the outage probability approach one regardless of the number of antennas used and noise characteristics.

\section{CONCLUSION}

In this paper we evaluated the ergodic outage probability performance of a WPNC system in the presence of nonGaussian noise. The system model considered in this work consists of an AP equipped with multiple-antennas and an energy-constrained node with a single-antenna. An analytical expression for the ergodic outage probability was derived and then verified with computer simulations. It was shown that optimizing the energy harvesting time is important to achieve best performance. Results also revealed that increasing the 


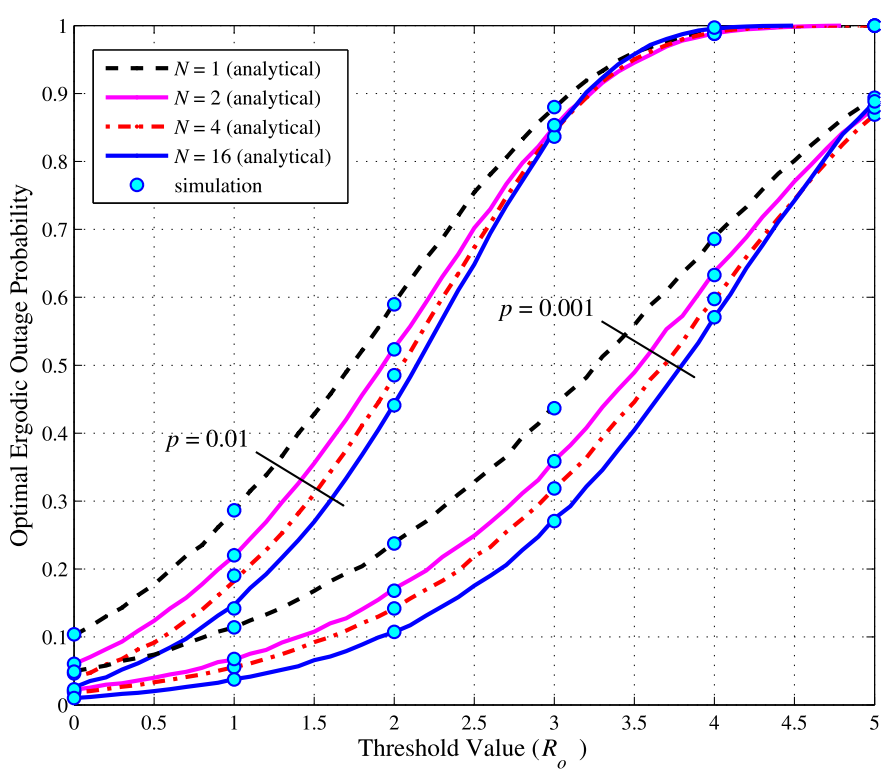

Figure 4: Optimal ergodic outage probability performance versus the threshold value for various values of $N$ and $p$.

number of AP antennas and/or source transmit power can improve the system performance.

\section{ACKNOWLEDGMENT}

This work was funded by Innovate UK as part of the "Smart In-building Micro Grid for Energy Management" (Innovate UK project 101836).

\section{REFERENCES}

[1] X. Zhou, R. Zhang, and C. K. Ho, "Wireless information and power transfer: Architecture design and rate-energy tradeoff," IEEE Trans. Commun., vol. 61, pp. 4754-4767, Nov. 2013.

[2] C. K. Ho and R. Zhang, "Optimal energy allocation for wireless communications with energy harvesting constraints," IEEE Trans. Signal Process., vol. 60, pp. 4808-4818, Sept. 2012.

[3] S. Lee, R. Zhang, and K. Huang, "Opportunistic wireless energy harvesting in cognitive radio networks," IEEE Trans. Wireless Commun. vol. 12, pp. 4788-4799, Sept. 2013.

[4] H. Ju and R. Zhang, "Throughput maximization in wireless powered communication networks," IEEE Wireless Commun., vol. 13, pp. 418 428, Jan. 2014

[5] K. Huang and E. Larsson, "Simultaneous information and power transfer for broadband wireless systems," IEEE Trans. Signal Process., vol. 61, pp. 5972-5986, Dec. 2013.

[6] A. Nasir, X. Zhou, S. Durrani, and R. Kennedy, "Relaying protocols for wireless energy harvesting and information processing," IEEE Wireless Commun., vol. 12, pp. 3622-3636, Jul. 2013
7] I. Krikidis, S. Timotheou, and S. Sasaki, "RF energy transfer for cooperative networks: Data relaying or energy harvesting?," IEEE Commun. Lett., vol. 16, pp. 1772-1775, Nov. 2012.

[8] H. Chen, Y. Li, J. Luiz Rebelatto, B. Uchoa-Filho, and B. Vucetic, "Harvest-then-cooperate: Wireless-powered cooperative communications," IEEE Trans. Signal Process., vol. 63, pp. 1700-1711, Apr. 2015.

[9] R. Zhang and C. K. Ho, "MIMO broadcasting for simultaneous wireless information and power transfer," IEEE Trans. Wireless Commun., vol. 12, pp. 1989-2001, May 2013.

[10] Z. Zhou, M. Peng, Z. Zhao, and Y. Li, "Joint power splitting and antenna selection in energy harvesting relay channels," IEEE Signal Process. Lett., vol. 22, pp. 823-827, Jul. 2015.

[11] Y.-C. Liang, Y. Zeng, E. Peh, and A. T. Hoang, "Sensing-throughput tradeoff for cognitive radio networks," IEEE Trans. Wireless Commun., vol. 7, pp. 1326-1337, Apr. 2008.

[12] S. Jafar and S. Srinivasa, "Capacity limits of cognitive radio with distributed and dynamic spectral activity," IEEE J. Select. Areas in Commun., vol. 25, pp. 529-537, Apr. 2007.

[13] K. Blackard, T. Rappaport, and C. Bostian, "Measurements and models of radio frequency impulsive noise for indoor wireless communications," IEEE J. Select. Areas in Commun., vol. 11, pp. 991-1001, Sept. 1993.

[14] D. Middleton, "Statistical-physical models of electromagnetic interference," IEEE Trans. Electromagn. Compat., vol. EMC-19, pp. 106-127, Aug. 1977.

[15] K. M. Rabie, E. Alsusa, and A. Salem, "Wireless power transfer over non-Gaussian channels with multiple-antenna access point," in Proc. IEEE Global Commun. Conf. (GLOBECOM), pp. 1-6, Dec. 2015.

[16] D. Middleton, "Canonical and quasi-canonical probability models of class a interference," IEEE Trans. Electromagn. Compat., vol. EMC25, pp. 76-106, May 1983.

[17] K. Wiklundh, P. Stenumgaard, and H. Tullberg, "Channel capacity of Middleton's class A interference channel," Electronics Letters, vol. 45 pp. 1227-1229, Nov. 2009.

[18] A. Kenarsari-Anhari and L. Lampe, "Performance analysis for BICM transmission over Gaussian mixture noise fading channels," IEEE Trans. Commun., vol. 58, pp. 1962-1972, Jul. 2010

[19] M. Ghosh, "Analysis of the effect of impulse noise on multicarrier and single carrier QAM systems," IEEE Trans. Commun., vol. 44 pp. 145-147, Feb. 1996.

[20] R. Pighi, M. Franceschini, G. Ferrari, and R. Raheli, "Fundamental performance limits of communications systems impaired by impulse noise," IEEE Trans. Commun., vol. 57, pp. 171-182, Jan. 2009.

[21] K. M. Rabie and E. Alsusa, "Performance analysis of adaptive hybrid nonlinear preprocessors for impulsive noise mitigation over power-line channels," in Proc. IEEE Int. Conf. Commun. (ICC), pp. 728-733, Jun 2015.

[22] K. Rabie and E. Alsusa, "Preprocessing-based impulsive noise reduction for power-line communications," IEEE Trns. Power Del., vol. 29 , pp. 1648-1658, Aug. 2014.

[23] H. Vu, N. Tran, T. Nguyen, and S. Hariharan, "Estimating shannon and constrained capacities of Bernoulli-Gaussian impulsive noise channels in rayleigh fading," IEEE Trans. Commun., vol. 62, pp. 1845-1856, Jun. 2014.

[24] S. M. ROSS, Introduction to probability models. 10th ed. Harcourt Academic Press, 2010. 